\title{
BioTracker: Ferramenta para rastrear larvas de polvos
}

Paulo H. L. Carlos ${ }^{1}$, João Paulo Trindade ${ }^{1}$, Anderson Abner de S. Souza ${ }^{1}$, Sylvia Lima de Souza Medeiro ${ }^{2}$, Sidarta Ribeiro ${ }^{2}$, Wilfredo Blanco ${ }^{1}$

'Departamento de Computação - Universidade do Estado do Rio Grande do Norte (UERN)

Instituto do Cérebro da UFRN

Av. Dr. João Medeiros Filho 59.120-200 - 3419 - Potengi - Natal - RN - Brasil paulo_lopesllehotmail.com, joaopaulo_09@hotmail.com, and.abner@gmail.com, sylvia.lsmedeirosegmail.com, sidartaribeiro@neuro.ufrn.br, wblancofegmail.com

Resumo. No campo da biologia, o registro de comportamentos de espécies é tipicamente feito de maneira manual, causando desconforto, imprecisões e dispêndio de tempo. Nessa perspectiva, o objetivo deste trabalho é desenvolver um software que utiliza técnicas de visão computacional para rastrear a locomoção de animais. O programa computacional foi desenvolvido à partir da observação de larvas de Polvos Insularis, uma espécie endêmica nas águas rasas da costa e ilhas oceânicas do norte e nordeste brasileiro. A ferramenta foi desenvolvida na linguagem de programação Python com auxílio da biblioteca código aberto OpenCV. Esta ferramenta permitiu rastrear, em tempo real, a localização das larvas (5-10 animais) com mais de $90 \%$ de precisão nos vídeos processados.

\begin{abstract}
In the area of biology, the behavior of species is typically recorded using a manual method, which can be uncomfortable, inaccurate and timeconsuming. In this perspective, the objective of this work is to develop a software that uses computer vision techniques to track animal locomotion. The computational program was developed through the observation of larvae of Octopus Insularis, an endemic species to the shallow waters of the coast and oceanic islands of northern and northeastern regions of Brazil. The tool was developed in the Python programming language and using the OpenCV open source library. This tool was able to trace, in real time, the location of the larvae (5-10 animals) with more than 90\% accuracy in the processed videos.
\end{abstract}

\section{Introdução}


Tendo em vista que há uma tendência na aquicultura mundial para a criação de polvos em cativeiros, e também considerando seu elevado preço no mercado, pesquisadores desta área estão concentrando esforços na intenção de construir avanços tecnológicos afim de atender essa demanda (Lenz, 2015). A espécie Octopus Insularis, são polvos comuns nas águas rasas do litoral das regiões Norte e Nordeste Brasileira. No arquipélago de Fernando de Noronha, por exemplo, a pesca dessa espécie tem sido uma das principais fontes de renda dos pescadores, além de ser uma alternativa a pesca de lagosta. A Figura 1 retrata a anatomia desta espécie no período larvário.

Figura 1. Anatomia da larva Octopus Insularis. (A) llustração esquemática de partes e dimensões do corpo. (B) llustração esquemática dos cromatóforos.

A

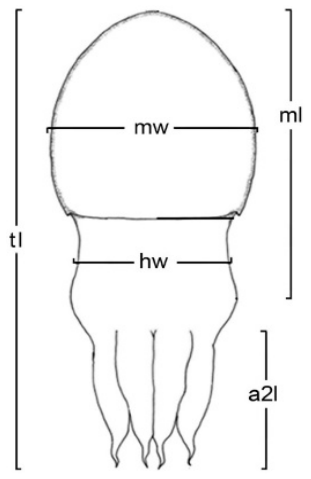

Ilustração mostrando as dimensões do corpo de uma larva Octopus insularis. Abreviações: a21, tamanho do segundo par braços; ed, diâmetro do olho; f1, tamanho do sifão; hw, largura cabeça; ml, largura do manto; tl, tamanho total.
$\mathrm{B}$

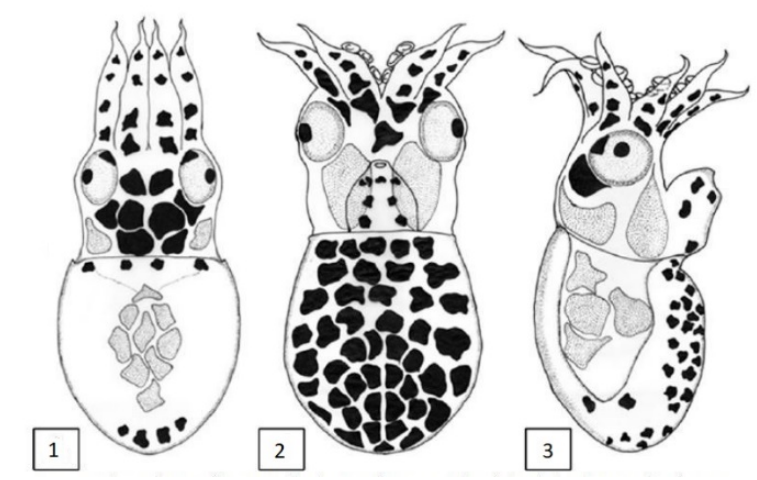

Octopus insularis. Ilustração esquemática dos padrões de cromatóforos de larvas recém eclodidas. 1, vista dorsal; 2, vista ventral; 3 , disposição lateral.

Fonte: adaptada de Lenz 2015

Através de analises visuais, constatamos quatro principais tipos de comportamento desta espécie. O comportamento nado Normal é o padrão do animal, ele fica alinhado de forma paralela ao chão com os olhos (Figura 1A,ed) virados para cima (Figura 1B1 e vídeo frame em Figura 2A), sendo esta a posição que a larva passa a maior parte do tempo. No nado em Pé (Figura 2B), o animal direciona seus braços para o chão e o saco/manto (Figura $1 \mathrm{~A}, m l$ ) para cima, simulando uma caminhada. Nado Lateral (Figura 1B3 e vídeo frame em Figura 2C), é similar ao Nado Normal, porém girando seu corpo noventa graus horizontalmente em torno do próprio eixo; nesta posição, apenas um olho é visível sobre a perspectiva superior. No comportamento Cleaning (Figura 2D), a larva se contrai inteira, deixando os braços junto ao saco/manto. Em todos esses comportamentos a larva pode ficar parada ou se movimentar pelo recipiente.

Figura 2. Comportamento das larvas do polvo Octopus Insularis

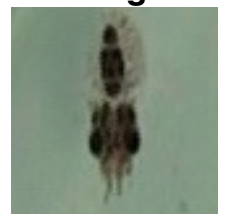

A - Normal

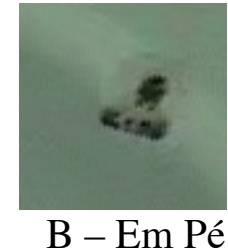

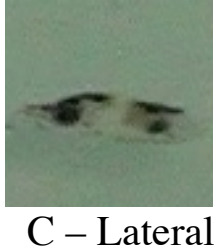

C - Lateral

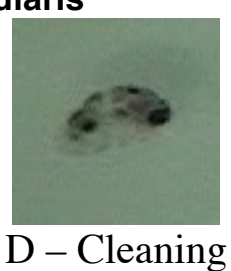

Em colaboração com UFRN (Programas de Pós-Graduação em Bioinformática - PPg de BioInfo e PPg em Neurociências) tivemos acesso a registos em vídeos do comportamento natural desses animais. Nesse contexto, desenvolvemos uma ferramenta 
para rastrear a localização em tempo real desta espécie no estágio larvário; facilitando o trabalho antes feito manualmente.

\section{Metodologia}

Para analisar o comportamento das larvas do Octopus Insularis Polvos, tivemos acesso a vídeos com aproximadamente 30 minutos de duração. Cada vídeo possui frames de resolução 1440x1080 e são gravações do comportamento de larvas que medem de 2 a 3 milímetros de cumprimento e estão num recipiente de 8 centímetros de diâmetro e 5 milímetros de profundidade.

\section{Programação e Desenvolvimento}

Para o desenvolvimento da ferramenta foi utilizado a Linguagem de Programação Python (Van Rossum, 2007) na versão 3.6.5 e a biblioteca Open Source OpenCV (Bradsky, 2006) na versão 3.4.1.

Visualmente percebemos que as larvas possuem coloração maiormente obscura, variando entre um valor inicial preto (zero) e um valor superior cinza (menor que 255). Para determinar este valor superior do intervalo utilizamos o Histograma (Figura 3); através dele constatamos que o melhor valor seria 80, pois desta forma conseguimos detectar as larvas em movimento e estática.

Figura 3. Histograma

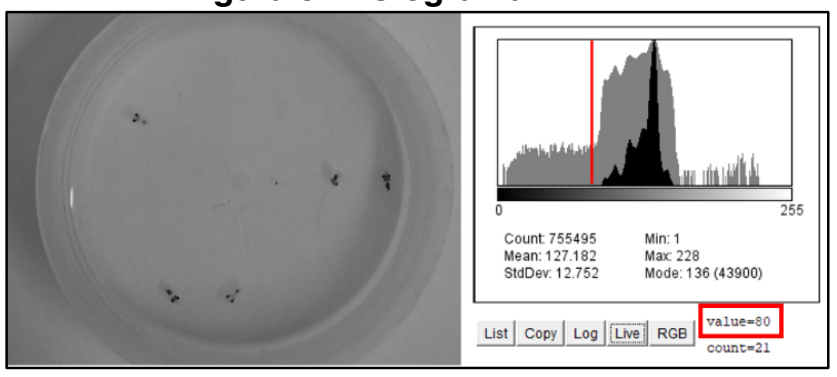

\section{Resultados}

Os vídeos são processados quadro a quadro (frames), e em cada frame realizamos uma sequencia de procedimentos (pipeline) para detectar e rastrear os animais (Figura 4).

Primeiro convertemos o frame colorido (sistema RGB) para a escala de cinza (Figura 4A, B Gray). Em seguida executamos uma binarização baseado no limiar previamente calculado; em outras palavras, geramos uma imagem só com cores branco e preto, onde todo pixel que sua cor original era menor que 80 passa a ser branco, e tudo que estava acima de 80 passa a ser preto (Figura 4B, Limiarização). Depois detectamos os contornos (Suzuki, 1985) das regiões brancas, retornando uma lista de coordenadas x,y. Em posse destas coordenadas, aplicamos o algoritmo K-Means (Kanungo, 2002) para detectar agrupamentos (clusters). Cada agrupamento de pontos passa a ser uma larva específica (Figura 4B, K-Means).

Figura 4: Pipeline de processamento. (A) Fluxo do dados e operações do pipeline. (B) Resultado das operações

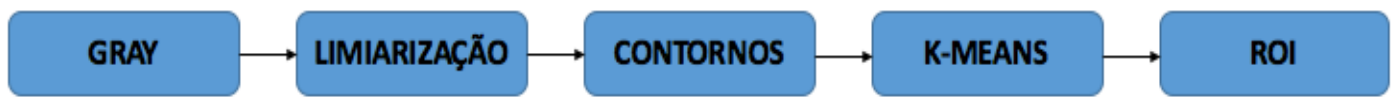


A

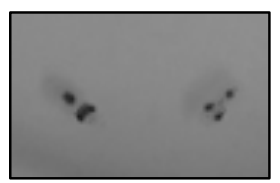

Gray

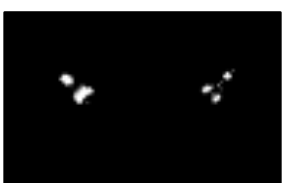

Limiarização

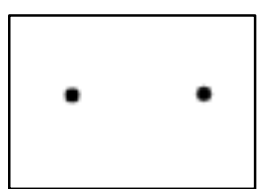

K-Means

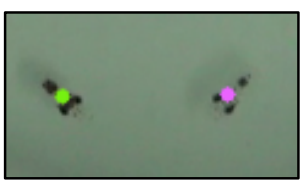

Rastreamento

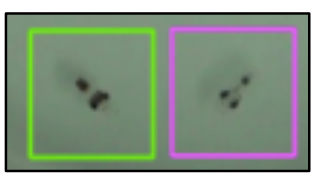

ROI

B

Para identificar e rastrear a larva implementamos um algoritmo simples, baseado na distância euclidiana mínima entre dois frames em sequência; i.e., reconhecemos que se trata do mesmo individuo/larva (clusters detectados pelo K-means), se o centro massa dele no frame atual e o subsequente tem a menor distância euclidiana. Por último, exibimos o frame de vídeo colorido original destacando um ponto colorido para cada indivíduo/larva e sua ROI (Region of Interest) correspondente de dimensão 80x80 pixels (Figura 4B, ROI). Este valor de dimensão foi calculado propositalmente para enquadrar a larva em todas as possíveis posições, evitando espaços desnecessários.

Com essa ferramenta foi possível rastrear em tempo real a localização de larvas (5 a 10 animais) com $90 \%$ de precisão nos vídeos processados. Até a elaboração deste trabalho não encontramos na literatura pesquisas similares que nos permita realizar algum tipo de estudo comparativo. É importante também mencionar que o trabalho ainda está em desenvolvimento, portanto, os parâmetros utilizados na execução de cada módulo, ainda estão sendo investigados, afim de se conseguir os melhores resultados.

\section{Conclusão}

Apresentamos o uma ferramenta robusta capaz de rastrear larvas de polvo da espécie Octopus Insularis Polvos com os objetivos principais: facilitar o estudo sobre este animal e automatizar o processo de detecção e rastreamento. Para isso, foram utilizadas técnicas de visão computacional no processo de desenvolvimento. Atualmente, estamos desenvolvendo um módulo para detectar e classificar os tipos de comportamentos previamente citados, para essa tarefa estamos usando redes neurais artificiais profundas especificamente as redes convolutivas (Convolutional Neural-Network).

\section{Referências}

BATISTA, Allan Torrecilla; LEITE, Tatiana Silva. Octopus insularis (Cephalopoda: Octopodidae) on the tropical coast of Brazil: where it lives and what it eats. Brazilian Journal of Oceanography, v. 64, n. 4, p. 353-364, 2016.

LENZ, Tiago de Moraes. Desenvolvimento de polvos Octopus spp. durante a fase planctônica: subsídios para o cultivo. 2015.

BRADSKY, G. R.; PISAREVSKY, V.; BOUGUET, J. Learning OpenCV: Computer Vision with the OpenCV Library. Springer, 2006.

LENZ, Tiago M. et al. First description of the eggs and paralarvae of the tropical octopus, Octopus insularis, under culture conditions. American Malacological Bulletin, v. 33, n. 1, p. 101-109, 2015. 
VAN ROSSUM, Guido et al. Python Programming Language. In: USENIX Annual Technical Conference. 2007. p. 36.

Kanungo, T., Mount, D. M., Netanyahu, N. S., Piatko, C. D., Silverman, R., \& Wu, A. Y. (2002). An efficient k-means clustering algorithm: Analysis and implementation. IEEE Transactions on Pattern Analysis \& Machine Intelligence, (7), 881-892.

Suzuki, S. (1985). Topological structural analysis of digitized binary images by border following. Computer vision, graphics, and image processing, 30(1), 32-46. 